\title{
An Improved Electric Model with Online Parameters Correction for Large Li-Ion Battery Packs
}

\author{
Daiming Yang, Chao Lu, and Guoguang Qi
}

\begin{abstract}
Lithium-ion battery packs based battery energy storage system (BESS) has proved its high potential in energy storage. In studying the BESS, A precise battery model is critical for implementing the system simulation, estimating the SOC, SOH of the battery, as well as optimizing the design. An electric model which can reflect the hysteresis effect and dynamic characteristic is adopted in this paper, and based on that, we proposed a novel method for battery model identification and on-line correction of model parameters by extended Kalman filter (EKF). Thus the parameters of battery model can be regulated in the progress of battery charge and discharge. The method is proved to be efficient and accurate by comparing the simulation result with the experiment data.
\end{abstract}

Index Terms-Battery modeling, Li-ion battery pack, extended Kalman filter (EKF), model identification, on-line correction.

\section{INTRODUCTION}

With the development of the lithium-ion battery technology, especially the promotion of electric vehicle research in recent years, the application of large lithium-ion battery energy storage system continues to expand, and the costs decline rapidly[1]. Battery model plays a vital role in the study of large-scale lithium battery energy storage technology. Commonly used battery model includes electrochemical model, circuit model and mathematical model. Meanwhile, the circuit model is not only the most thoroughly studied, but also the most widely used. It can be used for electrical system simulation, aiding system design, and it is also the necessity for developing BMS. The battery model can help us to estimate the state of charge (SOC), state of health $(\mathrm{SOH})$ such as power fade and capacity fade, calculate instantaneous available power, and predict remaining running time, as well as track electrical characteristic of battery[2], [3].

Decided by the chemical substances inside the battery, battery shows complex electrical characteristics. The model which is able to accurately reflect the electrical characteristics of the battery is complicated and nonlinear. The number of wanted unknown parameters in the model and the accuracy of the model are positive correlation. The more parameters contained, the higher precision of the model will

Manuscript received October 19, 2012; revised November 23, 2012. This work was supported in part by the National High Technology Research and Development Program of China (2011AA05A111).

D. Yang and C. Lu are with the Department of Electrical Engineering, Tsinghua University, Beijing 100084 China (e-mail: yangdaiming@ gmail.com, luchao@tsinghua.edu.cn).

G. Qi is with the Department of Computer Science, Tsinghua University, Beijing 100084 China (e-mail: qiguo@ mail.tsinghua.edu.cn). receive. However, the parameter identification algorithm is also more complicated, resulted in complex calculation. With the aging of the battery and the changing of temperature, the battery performance will change constantly, so do the model parameters. The model parameters got in a certain test, which are consistent with the actual characteristics of the battery, but may not work well in other conditions. Therefore, a battery model which could be able to change over test environment is needed.

The battery model that proposed in this paper is improved on the basis of the Thevenin circuit, in order to capture the nonlinear characteristics and dynamic characteristics. We took EKF method, which is an intelligent means for estimating state value of a dynamic system, as the model parameter identification algorithm [4-6].

In many research and applications, the model parameters are fixed. In fact, the circuit model cannot fully reflect performance of the battery, of which the parameters are needed to change with different battery states and ambient temperature, in order to adapt to the actual battery better. Especially, taking the Kalman filter or EKF algorithm to estimate the battery state, such as SOC, requires an accurate model. In order to get model parameters under different conditions, a large number of specific charge-discharge experiments have to be conducted, wherein the workload is quite large. Therefore, we studied an online method for parameters correction, which is able to fix the parameters of the original model when the energy storage system is running. For large-scale battery energy storage system, the method can achieve a goal that the system runs with model identification and parameters correction.

\section{Thevenin-Based Electrical Model}

The electrical model, which is showed in Fig. 1, is based on Thevenin circuit.

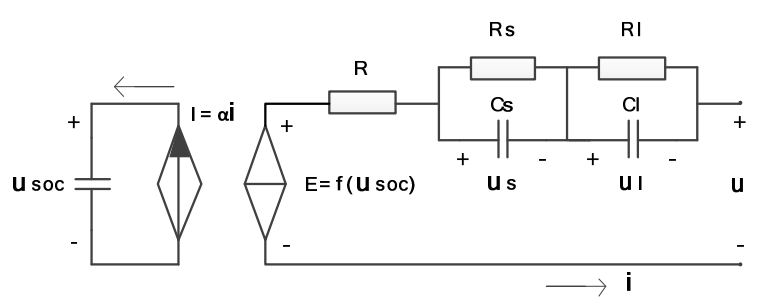

Fig. 1. Electrical model based on Thevenin circuit[7]

In this model, two parts are contacted by controlled sources. There are a current controlled current source (CCCS), which is controlled by the current flowing in/out of the battery, and a capacitor $\left(C_{s o c}\right)$, of which the voltage $\left(u_{s o c}\right)$ range from $0 \mathrm{~V}$ to $1 \mathrm{~V}$ means SOC from $0 \%$ to $100 \%$. The right part is formed of a voltage controlled voltage source 
(VCVS), a resistor and two RC parallel networks. The VCVS is the electromotive force (EMF), which is controlled by SOC or $u_{\text {soc }}$. The resistor $\mathrm{R}$ characterizes instantaneous response, whereas the two RC parallel networks $\left(R_{s}, C_{s}, R_{\mathrm{l}}, C_{\mathrm{l}}\right)$ are for short-time and long-time transient response.

According to the model above, we can get the state equation (1) and the output equation (2)[8].

$$
\begin{aligned}
& {\left[\begin{array}{l}
u_{s o c}(k+1) \\
u_{c s}(k+1) \\
u_{c l}(k+1)
\end{array}\right]=\left[\begin{array}{ccc}
1 & 0 & 0 \\
0 & 1-\frac{\Delta t}{R_{s} \cdot C_{s}} & 0 \\
0 & 0 & 1-\frac{\Delta t}{R_{l} \cdot C_{l}}
\end{array}\right]\left[\begin{array}{l}
u_{s o c}(k) \\
u_{c s}(k) \\
u_{c l}(k)
\end{array}\right]} \\
& +\left[\begin{array}{c}
\frac{\alpha \Delta t}{C_{0}} \\
-\frac{\Delta t}{C_{s}} \\
-\frac{\Delta t}{C_{l}}
\end{array}\right] i(k) \\
& u(k)=E M F\left(u_{s o c}(k)\right)-u_{c s}(k)-u_{c l}(k)+R \cdot i(k)
\end{aligned}
$$

In most cases, batteries are not allowed to be charged to $100 \%$ or discharged to $0 \%$, so as to avoid over charge and over discharge, prolong life of batteries. So we only study the characteristic of Lithium-ion battery in the SOC range from $10 \%$ to $95 \%$. Therefore, the $\mathrm{u}_{\text {soc }}$ changes from $0.05 \mathrm{~V}$ to $0.95 \mathrm{~V}$.

\section{MODEL IDENTIFICATION}

\section{A. Electromotive Force}

As changing with different SOC, EMF is taken as a function of $\mathrm{u}_{\text {soc }}$, which is showed in Fig.1. Typically, there are two methods to determine EMF: linear interpolation and voltage relaxation. Both of them take quite a long time to get the curve of SOC-EMF. In this paper, we introduced a simpler method, which comprises the following steps. Firstly, a battery is charged from SOC $=0 \%$ to the upper limit voltage at a constant current, set as i0. Secondly, charge the battery at the upper limit voltage till the current drops to 0.01C. At the end of the charge step, the SOC is defined to be $100 \%$. Thirdly, a discharge step is applied at $-\mathrm{i}_{0}$ until the battery voltage reaches the lower limit voltage. Fourthly, discharge the battery at the lower voltage till the current drops to $0.01 \mathrm{C}$. At the end of the discharge step, the SOC is defined to be $0 \%$. From the first and third steps, two curves of SOC-u, in which SOC is calculated by current integration, can be obtained. We took the SOC-u curves instead of SOC-EMF. When the battery is charged at a current $i_{c}$, SOC-u curve in charge step is applied and the current is modified to $\mathrm{i}_{\mathrm{c}}-\mathrm{i}_{0}$. When the battery is discharged at a current id, SOC-u curve in discharge step is applied and the current is modified to $\mathrm{i}_{\mathrm{d}}+\mathrm{i}_{0}$.

When the state of battery changes from charge to discharge, or reverse. The hysteresis effect should be considered. The maximum hysteresis voltage is the height difference between charge curve and discharge curve. The change of hysteresis can be described as follow

$$
\dot{u}_{h}=\operatorname{sgn}(i) \cdot\left|u_{h \max }-u_{h}\right|
$$

The $u_{h}$ denotes the hysteresis voltage, which is decided by the battery state, changing with time, current and temperature. The $\mathrm{u}_{\mathrm{hmax}}$ is a function of SOC. When the current is not zero, the larger difference between the $u_{h \max }$ and the $u_{h}$, the more quickly $u_{h}$ changes. When the current is zero, the $u_{h}$ stays the same.

\section{B. Resistor $R$}

The resistor $\mathrm{R}$ is the $\mathrm{DC}$ resistance that reflects the changed voltage along with the current. $\mathrm{R}$ can be calculated by the instantaneous changed voltage and current.

$$
R=\frac{d u}{d i}
$$

Pulse charge (or discharge) experiments are performing to calculate R, with the result showed in Fig. 2. According to the result, the $\mathrm{R}$ is relatively stable from SOC $=5 \%$ to SOC $=95 \%$. Therefore, $\mathrm{R}$ can be considered as constant with different SOC.

\section{RC parallel circuit}

The dynamic characteristic of the battery is related to the RC parallel circuit. To simplify the circuit model, $\mathrm{R}$ and $\mathrm{C}$ do not changed with SOC, which can be applied to a variety of occasions.

There are two means to get RC parameters, one is pulse charge-discharge experiment across the whole range of SOC, the other is hybrid pulse power characterization (HPPC) test [9].

The first method is applied to calculate RC parameters by the least squares fit (LSF) algorithm, which is adopted in this paper.

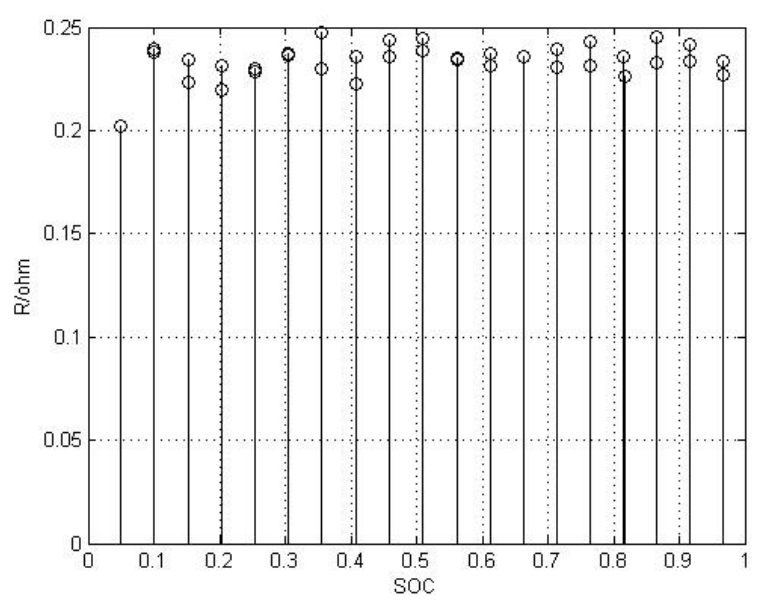

Fig. 2. Resistor R at different SOC

\section{PARAMETERS ON-LINE CORRECTION}

The EKF is used to correct parameters of a battery pack on-line. The algorithm combines two EKFs, one is for state estimating and the other is for parameters correcting [10]. 
The EKF for state estimating is running at every time step, ignoring the working condition. However, the EKF for parameters correcting is only running when it is needed or in special condition. This paper only takes the last EKF into research.

To perform model parameters correction, a state-space model is required. As the parameters changing very slowly, they can be modeled as constant with some perturbation, that is

$$
p(k+1)=p(k)+v(k)
$$

$v(k)$ is taken as noise input, which models the small change of the parameters to increase the accuracy of the system. $p(k)$ is the state vector, described as follow

$$
p(k)=\left[E M F(k), R_{s}(k), R_{l}(k), C_{s}(k), C_{l}(k)\right]^{T}
$$

Because $R$ can be calculated directly by (4), it is not taken as a state parameter.

The output equation of the system for the EKF is the same with (2). Whereas, a input modeled the measurement noise, setting as $w(k)$, is added.

In definition part above, $C(k)$ is derived as (7) showed. The first part in the left side is zero, and the second can be got by (1) and (2).

$$
\begin{aligned}
C(k) & =\left.\frac{d g(x(k), i(k), p)}{d p}\right|_{p=p(k \mid k-1)} \\
& =\frac{\partial g(x(k), i(k), p)}{\partial p}+\frac{\partial g(x(k), i(k), p)}{\partial x(k)} \cdot \frac{d x(k)}{d p}
\end{aligned}
$$

TABLE I: EKF FOR MODEL PARAMETERS CORRECTION

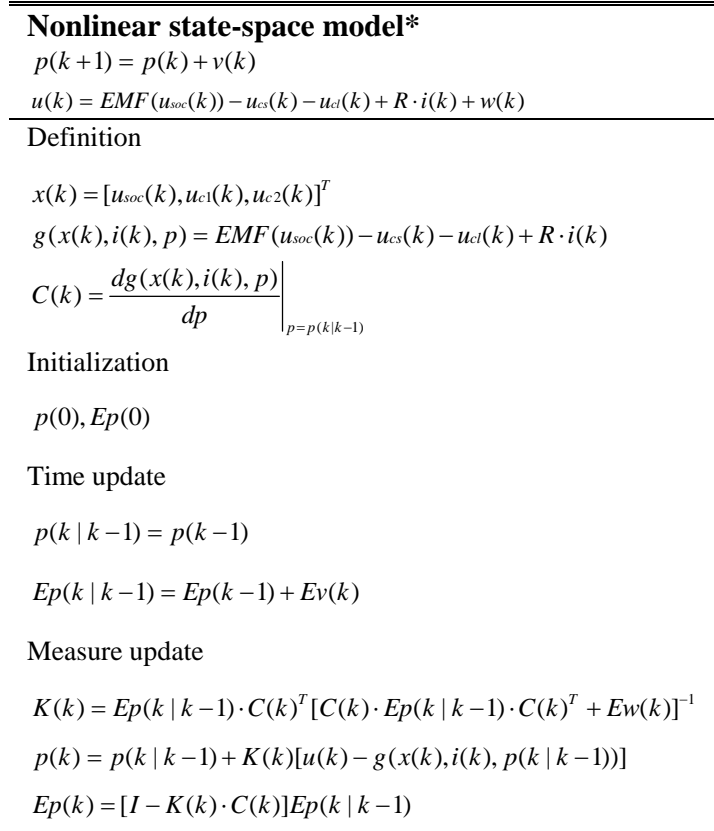

$\overline{* v(k) \text { and } w(k) \text { are system noise and measurement noise. } E v(k) \text { and } E w(k) \text { are }}$ the covariance matrices of them, respectively.

For some parameters coupling, not all the parameters can be corrected at the same time. Simulation result shows that a parameter can be identified in some conditions, while not in other conditions.
EMF can only be corrected when the current is approximately zero and the state is stable. In the other word, the output voltage does not change any more. The Rs and Rl can be corrected when the current stays constant or changes around a very small range. The $C_{s}$ and $C_{l}$ can be modified when the battery gets into relaxation state. In the state, the current drops to zero and the voltage increases or decreases slowly. What should be noticed is that $R_{s}$ and $R_{l}$ must stay constant in relaxation state and $\mathrm{C}_{\mathrm{s}}$ and $\mathrm{C}_{\mathrm{l}}$ can not be changed when the current is constant.

\section{EXPERIMENTAL AND SiMUlation RESUltS}

To verify the model identification and parameters correction algorithms, simulations have been performed, so do the experiments. The simulation tool is MATLAB, and a $\mathrm{m}$-file is created to implement the algorithm. The experiment platform is a $1 \mathrm{~kW} \quad \mathrm{LiFePO}_{4}$ battery energy storage experimental system, containing a 51.2V/20Ah $\mathrm{LiFePO}_{4}$ battery pack, an adjustable power supply, a controllable electric load and an embedded system for sampling, processing and communicating with a PC. The experimental data of battery cycling is collected through the embedded system and sent to the PC as inputs to the simulations.

Simulations and experiments are implemented as the following steps. First, the model identification is conducted with the methods described in Part 3. The identification algorithm is off-line, global optimum. Second, parameters got in first step are taken as the model initial parameters. Then the EKF for model state estimating starts to work. Third, to verify the EKF algorithm for correcting parameters in different conditions, we should change conditions. However, for the limit of experiment condition, we change the model parameters instead of changing the experiment environment. Fourth, the EKF algorithm is running for parameters correction.

Thus, we can get results from three conditions, one for initial parameters as the standard condition, one for changed parameters as the changed environment, the other for changed parameters with EKF parameters correction.

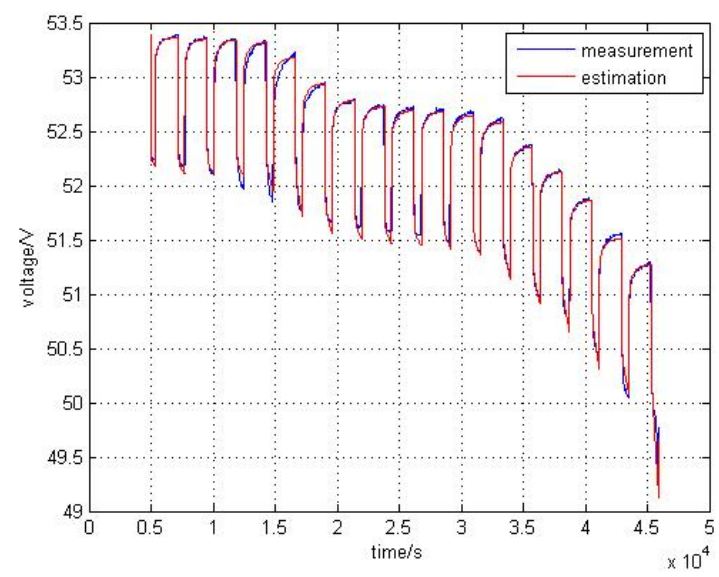

Fig. 3. Comparison between the measurement voltage and the simulation voltage got by the pulse discharge test from SOC $=5 \%$ to $95 \%$

A pulse discharge experiment from SOC $=5 \%$ to $95 \%$ has been performed. The output voltage is showed in Fig. 3, comparing the measurement data and the estimation result. 
Because results got by the three conditions above are slightly different with each other, no all the voltage comparisons are in the figure. However, the average voltage errors are listed in Table II.

TABLE II: AVERAGE VOLTAGES ERRORS

\begin{tabular}{lll}
\hline \hline Initial parameters & Parameters changed & parameters correction \\
\hline $55.2 \mathrm{mV}$ & $92.7 \mathrm{mV}$ & $72.8 \mathrm{mV}$ \\
\hline \hline
\end{tabular}

\section{SUMMARIES}

Based on the modified thevenin circuit model, an model identification method is proposed, so is an on-line EKF algorithm for model parameters correction. According to the simulation and experiment result, the model indentification method is effective. When the model parameters change, the EKF algorithm for on-line parameters correction works well, decreasing about $21.5 \%$ error compared with that without parameters correction.

\section{REFERENCES}

[1] K. C. Divya and J. Ostergaard, "Battery energy storage technology for power systems-An overview," Electric Power Systems Research, vol. 79, pp. 511-520, 2009.

[2] C. Min and G. A. Rincon-Mora, "Accurate electrical battery model capable of predicting runtime and $\mathrm{I}-\mathrm{V}$ performance," IEEE Transactions on Energy Conversion, vol. 21, pp. 504- 511, 2006-01-01 2006.

[3] N. A. Chaturvedi, R. Klein, J. Christensen, J. Ahmed, and A. Kojic, "Modeling, estimation, and control challenges for lithium-ion batteries," in Proc. American Control Conference (ACC), 2010, pp. 1997-2002.

[4] G. L. Plett, "Extended Kalman filtering for battery management systems of LiPB-based HEV battery packs - Part 1. Background," Journal of Power Sources, vol. 134, pp. 252-261, 2004.

[5] G. L. Plett, "Extended Kalman filtering for battery management systems of LiPB-based HEV battery packs - Part 2. Modeling and identification," Journal of Power Sources, vol. 134, pp. 262-276, 2004.

[6] G. L. Plett, "Extended Kalman filtering for battery management systems of LiPB-based HEV battery packs - Part 3. State and parameter estimation," Journal of Power Sources, vol. 134, pp. 277-292, 2004.

[7] R. C. Kroeze and P. T. Krein, "Electrical battery model for use in dynamic electric vehicle simulations," in Proc. IEEE Power Electronics Specialists Conference, 2008, pp. 1336-1342.
[8] L. Jianwei, M. Mazzola, J. Gafford, and N. Younan, "A new parameter estimation algorithm for an electrical analogue battery model," in Proc. Twenty-Seventh Annual IEEE Applied Power Electronics Conference and Exposition (APEC), 2012, pp. 427-433.

[9] H. Hongwen, X. Rui, Z. Xiaowei, S. Fengchun, and F. JinXin, "State-of-Charge Estimation of the Lithium-Ion Battery Using an Adaptive Extended Kalman Filter Based on an Improved Thevenin Model," in Proc. IEEE Transactions on Vehicular Technology, vol. 60, pp. 1461-1469, 2011-01-01 2011.

[10] S. Lee, J. Kim, J. Lee, and B. H. Cho, "State-of-charge and capacity estimation of lithium-ion battery using a new open-circuit voltage versus state-of-charge," Journal of Power Sources, vol. 185, pp. 1367-1373, 2008.

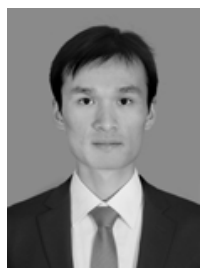

Daiming Yang was born in Yulin, Guangxi, China, on January 25, 1990. He received the B.S. degree from Tsinghua University, Beijing, China, in 2011. He is currently working toward the M.S. degree at Department of Electrical Engineering at Tsinghua University. Since November 2010, he has been studied on battery management system (BMS) for large lithium-ion battery packs in battery energy storage system (BESS). His research interests include battery modeling, SOC estimation and BMS design.

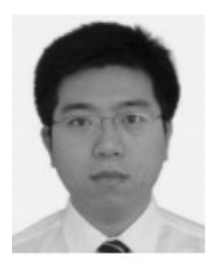

Chao Lu received the B.E. and Ph.D. degrees from Tsinghua University, Beijing, China, in 1999 and 2005, respectively. He is currently an associate professor with the Department of Electrical Engineering, Tsinghua University. His research interests include battery energy storage technology, power system analysis and control, and intelligent control applications.

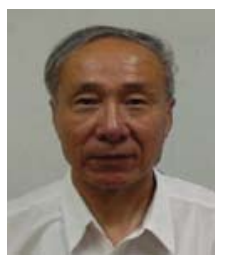

Guoguang Qi is a professor in the Department of Computer Science, Tsinghua University, Beijing, China. His research interests include computer control, artificial intelligence and battery management technology. He has been studying on electric vehicle (EV), hybrid electric vehicle (HEV) and battery storage technology for about 20 years. Since 1995, he has been responsible for the battery charging discharging management, battery management system and SOC estimation in several National Key Projects. 